PROCEEDINGS OF THE

AMERICAN MATHEMATICAL SOCIETY

Volume 140, Number 6, June 2012, Pages 2169-2178

S 0002-9939(2011)11056-6

Article electronically published on October 12, 2011

\title{
RELATIONS BETWEEN METRICS OF ALMOST POSITIVE CURVATURE ON THE GROMOLL-MEYER SPHERE
}

\author{
OWEN DEARRICOTT \\ (Communicated by Chuu-Lian Terng)
}

\begin{abstract}
We give a simplified proof that Wilhelm's metric on the GromollMeyer sphere has positive curvature almost everywhere. We determine that its zero locus coincides with that of the almost positively curved metric of Eschenburg and Kerin.
\end{abstract}

\section{INTRODUCTION}

$G=\operatorname{Sp}(2)=\left\{Q \in M_{2}(\mathbb{H}) \mid Q^{*} Q=I\right\}$ is endowed with a bi-invariant metric with non-negative curvature on which $H=\operatorname{Sp}(1)$ acts freely and isometrically by

$$
q \cdot Q \equiv q Q\left(\begin{array}{cc}
\bar{q} & 0 \\
0 & 1
\end{array}\right) .
$$

Gromoll and Meyer, 4, observed that the orbit space $M=G / / H$ is an exotic 7-sphere and inherits non-negative sectional curvature by O'Neill's formula, 5 . Although $M$ has points with strictly positive curvature, Wilhelm, [6], identified an open set in $M$ having zero curvature at each point and gave a different metric on this exotic 7-sphere with positive curvature almost everywhere. Subsequently Eschenburg and Kerin, 3, gave a clear proof that there was a second biquotient metric on $M=G / / H$ with almost positive curvature. We will see that these two almost positively curved metrics have the same zero curvature locus in diffeomorphism type.

By fully exploiting the machinery for biquotients we achieve a more concise and accessible proof that Wilhelm's metric has positive curvature almost everywhere than the original argument of [6. We give a description of the zero locus in this framework in Propositions 2.3, 2.4, 4.1, 4.2 and 4.3. Interest has been revived in this metric in connection with deformations of it proposed by Wilhelm and Petersen to put positive sectional curvature on this exotic 7-sphere.

\section{Preliminaries}

Proposition 1.1. Let $(G,\langle\rangle$,$) be a compact Lie group with a bi-invariant Rie-$ mannian metric. Then the sectional curvature of $G$ is non-negative. Moreover the zero planes at each point are precisely spanned by $x, y \in \mathfrak{g}$ with $[x, y]=0$.

Received by the editors November 10, 2010 and, in revised form, February 1, 2011.

2010 Mathematics Subject Classification. Primary 53C21.

(C)2011 American Mathematical Society Reverts to public domain 28 years from publication 
Definition 1.1. Consider the orbit space of a free isometric action of one compact Lie group, $H$, on another $(G,\langle\rangle$,$) , equipped with a bi-invariant metric defined via$ two representations, $\varrho_{L}, \varrho_{R}: H \rightarrow G$, by $h \cdot g \equiv \varrho_{L}(h) g \varrho_{R}(h)^{-1}$, via multiplication on the left and multiplication by the inverse on the right. We call such a Riemannian with its natural metric a biquotient, $G / / H$.

The above is a slight abuse of the standard terminology.

Proposition 1.2. A biquotient, $G / / H$, has non-negative curvature and has zero curvature at $H \cdot g$ if and only if $x, y \in \mathfrak{g}$ at $g$ span a plane normal to $H \cdot g$ and $[x, y]=0$.

Recall Cheeger's trick of [1].

Proposition 1.3. Let $K$ be a compact Lie group that acts isometrically on a Riemannian manifold, $M$, via $\cdot$. Then $K \times M$ carries an isometric action of $K$ by $\left(k(\tilde{k}, m)=\left(\tilde{k} k^{-1}, k \cdot m\right)\right.$ and $M$ is diffeomorphic to the orbit space, $K \times M / K$.

Proof. Consider maps, $f: M \rightarrow K \times M / K$ defined by $f(m)=K(1, m)$ and $g(K(\tilde{k}, m))=\tilde{k} m . g$ is well defined since $\tilde{k} \cdot m=\left(\tilde{k} k^{-1}\right) \cdot(k m)$. Moreover $g(f(m))=$ $g(K(1, m))=m$ and $f(g(K(\tilde{k}, m)))=f(\tilde{k} \cdot m)=K(1, \tilde{k} \cdot m)=K\left(\left(\tilde{k}^{-1}\right)^{-1}, \tilde{k}^{-1}\right.$. $(\tilde{k} \cdot m))=K(\tilde{k}, m)$. Hence $f$ and $g$ are inverses. Both maps are smooth, hence the diffeomorphism.

We use two variants of Cheeger's trick. A similar variant was used to construct examples with almost positive curvature in [7].

Proposition 1.4. Let $K$ be a compact Lie group that acts isometrically on a Lie group $G$ via two representations, $\varrho_{L}, \varrho_{R}: K \rightarrow G$, by $k \cdot g \equiv \varrho_{L}(k) g \varrho_{R}(k)^{-1}$, via multiplication on the left and multiplication by the inverse on the right. Moreover let $\varrho: H \rightarrow K$ be a representation so that the action $h \odot g \equiv \varrho(h) \cdot g$ is free. Then the orbit space of the action of $H \times K$ on $K \times G$ defined by $(h, k) \cdot(\tilde{k}, g) \equiv\left(\varrho(h) \tilde{k} k^{-1}, k \cdot g\right)$ is diffeomorphic to the biquotient, $G / / H$.

Proof. Consider maps

$$
\begin{array}{rrr}
f: G / / H \rightarrow K \times G / / H \times K, & f(H \odot g) \equiv H \times K \cdot(1, g), \\
\tilde{f}: K \times G / / H \times K \rightarrow G / / H, & \tilde{f}(H \times K \cdot(\tilde{k}, g)) \equiv H \odot(\tilde{k} \cdot g) .
\end{array}
$$

Verify that $f$ is well defined,

$$
\begin{aligned}
H \times K(1, h \odot g) & =H \times K(1, \varrho(h) \cdot g)=H \times K\left(\varrho\left(h^{-1}\right) 1\left(\varrho(h)^{-1}\right)^{-1}, \varrho(h)^{-1} \cdot(\varrho(h) \cdot g)\right) \\
& =H \times K(1, g) .
\end{aligned}
$$

Verify that $\tilde{f}$ is well defined,

$$
\begin{aligned}
H \odot((h, k) \cdot(\tilde{k}, g)) & =H \odot\left(\left(\varrho(h) \tilde{k} k^{-1}\right) \cdot(k \cdot g)\right)=H \odot(\varrho(h) \cdot(\tilde{k} \cdot g) \\
& =H \odot(h \odot(\tilde{k} \cdot g)=H \odot(\tilde{k} \cdot g) .
\end{aligned}
$$

Verify that $f$ and $\tilde{f}$ are mutual inverses,

$$
\begin{aligned}
\tilde{f}(f(H \odot g)) & =\tilde{f}(H \times K(1, g))=H \odot(1 \cdot g)=H \odot g, \\
f(\tilde{f}(H \times K \cdot(\tilde{k}, g) & =f(H \odot(\tilde{k} \cdot g))=H \times K \cdot(1, \tilde{k} \cdot g) \\
& =H \times K \cdot\left(1\left(\tilde{k}^{-1}\right)^{-1}, \tilde{k}^{-1} \cdot(\tilde{k} \cdot g)\right)=H \times K \cdot(\tilde{k}, g) .
\end{aligned}
$$

Moreover $f$ and $\tilde{f}$ are smooth. 
Proposition 1.5. Consider representations, $\varrho_{L}: K \rightarrow G, \varrho_{R}: H \rightarrow G$ and $\varrho:$ $H \rightarrow K$ such that the isometric group action defined by $h \odot g \equiv \varrho_{L}(\varrho(h)) g \varrho_{R}(h)^{-1}$ via multiplication on the left and multiplication by the inverse on the right is fixed point free. Then the orbit space of the action of $H \times H \times K$ on $H \times K \times G$ defined by $\left(h, h_{1}, k\right) \cdot(\tilde{h}, \tilde{k}, g) \equiv\left(h \tilde{h} h_{1}^{-1}, \varrho\left(h_{1}\right) \tilde{k} k^{-1}, \varrho_{L}(k) g \varrho_{R}(h)^{-1}\right)$ is diffeomorphic to the biquotient $G / / H$.

Proof. Consider maps

$$
\begin{array}{ll}
f: G / / H \rightarrow H \times K \times G / / H \times H \times K, & f(H \odot g) \equiv H \times H \times K \cdot(1,1, g), \\
\tilde{f}: H \times K \times G / / H \times H \times K \rightarrow G / / H, & \tilde{f}(H \times H \times K \cdot(\tilde{h}, \tilde{k}, g)) \equiv H \odot\left(\varrho_{L}(\varrho(\tilde{h}) \tilde{k}) g\right) .
\end{array}
$$

Verify that $f$ is well defined,

$$
\begin{aligned}
H \times H \times K \cdot(1,1, h \odot g) & =H \times H \times K \cdot\left(1,1, \varrho_{L}(\varrho(h)) \varrho_{R}(h)^{-1}\right) \\
& =H \times H \times K \cdot\left(h 1 h^{-1}, \varrho(h) 1 \varrho(h)^{-1}, \varrho_{L}(\varrho(h)) g \varrho_{R}(h)^{-1}\right) \\
& =H \times H \times K \cdot(h, h, \varrho(h)) \cdot(1,1, g)=H \times H \times K \cdot(1,1, g) .
\end{aligned}
$$

To verify that $\tilde{f}$ is well defined, note that

$$
\begin{gathered}
\left(h, h_{1}, k\right) \cdot(\tilde{h}, \tilde{k}, g)=\left(h \tilde{h} h_{1}^{-1}, \varrho\left(h_{1}\right) \tilde{k} k^{-1}, \varrho_{L}(k) g \cdot \varrho_{R}(h)^{-1}\right) \text { and thus } \\
H \odot\left(\varrho_{L}\left(\varrho\left(h \tilde{h} h_{1}^{-1}\right) \varrho\left(h_{1}\right) \tilde{k} k^{-1}\right) \varrho_{L}(k) g \varrho_{R}(h)^{-1}\right)=H \odot\left(\varrho_{L}(\varrho(h) \tilde{k}) g \varrho_{R}(h)^{-1}\right) \\
=H \odot(h \odot g)=H \odot g .
\end{gathered}
$$

Verify that $f$ and $\tilde{f}$ are mutual inverses,

$$
\begin{aligned}
\tilde{f}(f(H \odot g)) & =\tilde{f}(H \times H \times K(1,1, g))=H \odot g \text { and } \\
f(\tilde{f}(H \times H \times K \cdot(\tilde{h}, \tilde{k}, g))) & =f\left(H \odot\left(\varrho_{L}(\varrho(\tilde{h}) \tilde{k}) g\right)\right)=H \times H \times K \cdot\left(1,1, \varrho_{L}(\varrho(\tilde{h}) \tilde{k}) g\right) \\
& =H \times H \times K \cdot\left(1, \tilde{h}^{-1},(\varrho(\tilde{h}) \tilde{k})^{-1}\right) \cdot\left(1,1, \varrho_{L}(\varrho(\tilde{h}) \tilde{k}) g\right) \\
& =(H \times H \times K) \cdot(\tilde{h}, \tilde{k}, g) .
\end{aligned}
$$

Moreover $f$ and $\tilde{f}$ are smooth.

\section{WilHELM'S METRIC}

Let $G=\operatorname{Sp}(2), K=\operatorname{Sp}(1)^{4}$ with $\varrho_{L}((p, q, r, s))=\operatorname{diag}(p, q)$ and $\varrho_{R}((p, q, r, s))=$ $\operatorname{diag}(r, s)$. Consider the bi-invariant metric on $K$ given by $\ell_{1}^{2}\langle,\rangle \oplus \ell_{2}^{2}\langle,\rangle \oplus \nu_{1}^{2}\langle,\rangle \oplus$ $\nu_{2}^{2}\langle$,$\rangle , where \langle$,$\rangle is standard on \operatorname{Sp}(1)$ and $\left\langle M, M^{\prime}\right\rangle^{\prime}=\operatorname{Re}\left(\operatorname{tr}\left(M^{*} M^{\prime}\right)\right)$ on $G$. Let $H=\operatorname{Sp}(1)$ and $\varrho(q)=(q, q, q, 1)$. Proposition 1.4 gives that the orbit space of $K \times G / / H \times K$ is diffeomorphic to the exotic 7 -sphere, $M=G / / H$. In [6] an isometric metric was realised by applying Proposition 1.3 to $M=G$ and submersing to $G / / H$. The isometry arises by virtue of the fact that the actions of $H \times 1$ and $1 \times K$ commute on $K \times G$.

2.1. Horizontal vectors. With no loss of generality, restrict to coset representatives, $P=((1,1,1,1), Q) \in K \times G$. Let $((\alpha, \beta, \gamma, \delta), M) \in \mathfrak{k} \oplus T_{Q} G=T_{P}(K \times G)$. Recall that

$$
\mathfrak{g}=\mathfrak{s p}(2)=\left\{\left(\begin{array}{rr}
u & x \\
-\bar{x} & v
\end{array}\right): u, v \in \operatorname{Im}(\mathbb{H}), x \in \mathbb{H}\right\}
$$


Define $M_{L}, M_{R} \in \mathfrak{g}$ via

$$
M=\left(l_{Q}\right)_{*}\left(M_{L}\right)=Q\left(\begin{array}{cc}
u_{L} & x_{L} \\
-\bar{x}_{L} & v_{L}
\end{array}\right)=\left(r_{Q}\right)_{*}\left(M_{R}\right)=\left(\begin{array}{cc}
u_{R} & x_{R} \\
-\bar{x}_{R} & v_{R}
\end{array}\right) Q .
$$

Proposition 2.1. The horizontal distribution at $((1,1,1,1), Q)$ is

$$
\mathcal{H}=\left\{\left(\ell_{1}^{-2} u_{R}, \ell_{2}^{-2} v_{R},-\nu_{1}^{-2} u_{L},-\nu_{2}^{-2} v_{L}, M\right) \in \mathfrak{k} \oplus T_{Q} G: u_{L}=u_{R}+v_{R}\right\} .
$$

Proof. Let $\epsilon \in \operatorname{Im}(\mathbb{H})$ be arbitrary and $Z=\left((\alpha, \beta, \gamma, \delta),\left(r_{Q}\right)_{*}\left(M_{R}\right)\right)$ be horizontal.

$Z$ is perpendicular to the fundamental field of $(0,(\epsilon, 0,0,0))$.

So $\ell_{1}^{2}\langle\alpha,-\epsilon\rangle+\left\langle\operatorname{diag}(\epsilon, 0), M_{R}\right\rangle^{\prime}=0$, i.e., $-\ell_{1}^{2}\langle\alpha, \epsilon\rangle+\left\langle\epsilon, u_{R}\right\rangle=0$. Hence $\alpha=$ $\ell_{1}^{-2} u_{R}$. Likewise consider that $Z$ is perpendicular to fundamental fields of $(0,(0, \epsilon, 0,0,0)),(0,(0,0, \epsilon, 0))$, and $(0,(0,0,0, \epsilon))$ in turn to deduce $\beta=\ell_{2}^{-2} v_{R}$, $\gamma=-\nu_{1}^{-2} u_{L}$ and $\delta=-\nu_{2}^{-2} v_{L}$.

$Z$ is perpendicular to the fundamental field of $(\epsilon,(0,0,0,0)) \perp((\alpha, \beta, \gamma, \delta), M)$. Hence $(\epsilon, \epsilon, \epsilon, 0,0) \perp((\alpha, \beta, \gamma, \delta), M)$. So $\ell_{1}^{2}\langle\alpha, \epsilon\rangle+\ell_{2}^{2}\langle\beta, \epsilon\rangle+\nu_{1}^{2}\langle\gamma, \epsilon\rangle=0$. Hence $\ell_{1}^{2} \alpha+\ell_{2}^{2} \beta+\nu_{1}^{2} \gamma=0$. Thus $u_{L}=u_{R}+v_{R}$.

2.2. Reducing the number of planes. It follows from Proposition 1.2 that zero planes, $\operatorname{span}\left\{M^{1}, M^{2}\right\}$, are characterized by the conditions $\left[u_{R}^{1}, u_{R}^{2}\right]=0,\left[v_{R}^{1}, v_{R}^{2}\right]=$ $0,\left[u_{L}^{1}, u_{L}^{2}\right]=0,\left[v_{L}^{1}, v_{L}^{2}\right]=0$ and $\left[M_{R}^{1}, M_{R}^{2}\right]=0$.

To begin consider the 3 conditions, $\left[u_{R}^{1}, u_{R}^{2}\right]=0,\left[v_{R}^{1}, v_{R}^{2}\right]=0$ and $\left[M_{R}^{1}, M_{R}^{2}\right]=0$; cf. [2]. The top left entry of $\left[M_{R}^{1}, M_{R}^{2}\right]$ is $\left[u_{R}^{1}, u_{R}^{2}\right]-x_{R}^{1} \bar{x}_{R}^{2}+x_{R}^{2} \bar{x}_{R}^{1}$; since $\left[u_{R}^{1}, u_{R}^{2}\right]=0$ and $\left[M_{R}^{1}, M_{R}^{2}\right]=0$, it follows that $\operatorname{Im}\left(x^{2} \bar{x}^{1}\right)=0$. That is, $x_{R}^{2} \bar{x}_{R}^{1} \in \mathbb{R} ;$ namely, $x_{R}^{1}$ and $x_{R}^{2}$ are linearly dependent. So without loss of generality, by taking a linear combination of $M^{1}, M^{2}$, one may assume that $x_{R}^{1}=0$. Put $x_{R}^{2}=x \in \mathbb{H}$ and put $u_{R}^{1}=u$ and $v_{R}^{1}=v$. The top right entry of $\left[M_{R}^{1}, M_{R}^{2}\right]$ is $u x-x v$; hence $\left[M^{1}, M^{2}\right]=0$ gives $u x-x v=0$. This splits into two cases: (1) $x=0$ or (2) $x \neq 0$ and $u=x v x^{-1}$.

(1) If $x=0$, then one may assume without loss that $M_{R}^{1}=\operatorname{diag}(u, 0)$ and $M_{R}^{2}=\operatorname{diag}(0, v)$ since conditions $\left[u_{R}^{1}, u_{R}^{2}\right]=0$ and $\left[v_{R}^{1}, v_{R}^{2}\right]=0$ give that $u_{R}^{1}, u_{R}^{2}$ and $v_{R}^{1}, v_{R}^{2}$ are linearly dependent.

(2) On the other hand if $x \neq 0$ and $u=x v x^{-1}$, then since $M^{1} \neq 0, u \neq 0$ one may assume without loss of generality that $u_{R}^{2}=0$. The linear dependence of $v$ and $v_{R}^{2}$ give $v_{R}^{2}=t v$ for some $t \in \mathbb{R}$. Hence without loss one may assume that $M_{R}^{1}=\operatorname{diag}\left(x v x^{-1}, v\right)$ and $M_{R}^{2}=\left(\begin{array}{rr}0 & x \\ -\bar{x} & t v\end{array}\right)$.

It suffices to consider cases (1) and (2) below:

$$
\begin{array}{ll}
M_{R}^{1}=\operatorname{diag}(u, 0), & M_{R}^{2}=\operatorname{diag}(0, v), \\
M_{R}^{1}=\operatorname{diag}\left(x v x^{-1}, v\right), & M_{R}^{2}=\left(\begin{array}{rc}
0 & x \\
-\bar{x} & t v
\end{array}\right) .
\end{array}
$$

2.3. Horizontal zero planes. Assume the zero plane, $\operatorname{span}\left\{M_{R}^{1}, M_{R}^{2}\right\}$, is horizontal; then $u_{L}^{1}=u_{R}^{1}+v_{R}^{1}$ and $u_{L}^{2}=u_{R}^{2}+v_{R}^{2}$. In case (11) it follows from the above that $u_{L}^{1}=u+0=u$ and $u_{L}^{2}=0+v=v$. Recall that $M_{L}=\operatorname{Ad}\left(Q^{-1}\right) M_{R}$. Let $Q=\left(\begin{array}{ll}a & b \\ c & d\end{array}\right)$; thus $u_{L}^{1}=\bar{a} u a$ and $u_{L}^{2}=\bar{c} v c$. Hence $\bar{a} u a=u$ and $\bar{c} v c=v$. Thus $a= \pm 1$ and $c= \pm 1$, a contradiction, since $Q \in \operatorname{Sp}(2)$. Hence no horizontal zero planes arise for (11).

For (2) consider the two remaining conditions for zero curvature, $\left[u_{L}^{1}, u_{L}^{2}\right]=0$ and $\left[v_{L}^{1}, v_{L}^{2}\right]=0$. The horizontal condition gives $u_{L}^{1}=x v x^{-1}+v$ and $u_{L}^{2}=t v$. 
Hence the condition $\left[u_{L}^{1}, u_{L}^{2}\right]=t\left[x v x^{-1}, v\right]=0$ splits into two cases, (i) $t=0$ and (ii) $t \neq 0$. Hence

Proposition 2.2. Every zero plane in $\mathcal{H}_{(e, Q)}$ may be written in the form described in Proposition 2.3 or Proposition 2.4 .

2.4. The $t=0$ case. Throughout, $\hat{x}$ denotes the unit length quaternion, $\frac{x}{|x|}$, for $x \neq 0$.

Proposition 2.3. $Q \in \mathrm{Sp}(2)$ has zero planes, $\operatorname{span}\left\{\left(*, M^{1}\right),\left(*, M^{2}\right)\right\} \subset \mathcal{H}_{(e, Q)}$, of the form

$$
M_{R}^{1}=\operatorname{diag}\left(x v x^{-1}, v\right), \quad M_{R}^{2}=\left(\begin{array}{cc}
0 & x \\
-\bar{x} & 0
\end{array}\right)
$$

where

$$
p v \bar{p}=q v \bar{q}+v
$$

for appropriately chosen $p, q \in \mathrm{Sp}(1)$. Namely

(i) if $a \bar{c} \neq 0,|\operatorname{Im}(\hat{c})| \geq \frac{1}{2}$ and $|\operatorname{Im}(\widehat{a \bar{c}})| \geq \frac{\sqrt{3}}{2}$, pick $p=\hat{c}^{-1}$ and $q=\widehat{a \bar{c}}$ and $x=a \bar{c}$

(ii) if $a=0$ and $|\operatorname{Im}(c)| \geq \frac{1}{2}$, pick $p=\bar{c}$ and $q=\hat{x}$ for some $x$ with $|\operatorname{Im}(\hat{x})| \geq$ $\frac{\sqrt{3}}{2}$

(iii) if $c=0$ and $|\operatorname{Im}(a)| \geq \frac{1}{2}$, pick $p=\bar{a} \hat{x}$ and $q=\hat{x}$ for some $x$ with $|\operatorname{Im}(\hat{x})| \geq$ $\frac{\sqrt{3}}{2}$.

A solution $(p, q, v)$ for (3) must have

(a) $v$ is a scalar multiple of $\operatorname{Im}(\bar{q} p q) \times \operatorname{Im}(p \bar{q})$ provided this quaternion is nonzero,

(b) $v$ is a scalar multiple of $\operatorname{Im}(q) \times \operatorname{Im}(\bar{q} p q)$ if $\operatorname{Im}(\bar{q} p q) \times \operatorname{Im}(p \bar{q})=0$ provided $\operatorname{Im}(p)$ and $\operatorname{Im}(q)$ are linearly independent,

(c) if $\operatorname{Im}(p)$ and $\operatorname{Im}(q)$ are linearly dependent, then $p=e^{\alpha \frac{\pi}{6}}$ and $q= \pm e^{\alpha \frac{\pi}{3}}$ for some purely imaginary unit quaternion, $\alpha$, with $v \perp \alpha$.

Proof. It follows that a plane of form (2) with $t=0$ has

$$
M_{L}^{2}=\operatorname{Ad}\left(Q^{-1}\right)\left(\begin{array}{cc}
0 & x \\
-\bar{x} & 0
\end{array}\right)=\left(\begin{array}{cc}
\bar{a} x c-\bar{c} \bar{x} a & * \\
* & \bar{b} x d-\bar{d} x b
\end{array}\right) .
$$

Thus the horizontal condition gives $u_{L}^{2}=2 \operatorname{Im}(\bar{a} x c)=0$. Hence $\bar{a} x c$ is real, so either $a \bar{c}=-b \bar{d}=0$ or $x$ is a scalar multiple of $a \bar{c}=-b \bar{d}$. In either case $v_{L}^{2}=2 \operatorname{Im}(\bar{b} x d)=$ 0 . So $\left[u_{L}^{1}, u_{L}^{2}\right]=\left[v_{L}^{1}, v_{L}^{2}\right]=0$ and

$$
M_{L}^{1}=\operatorname{Ad}\left(Q^{-1}\right)\left(\begin{array}{cc}
x v x^{-1} & 0 \\
0 & v
\end{array}\right)=\left(\begin{array}{cc}
\bar{a} x v x^{-1} a+\bar{c} v c & * \\
* & \bar{b} x v x^{-1} b+\bar{d} v d
\end{array}\right) .
$$

Hence the horizontal equation is $\bar{a} x v x^{-1} a+\bar{c} v c=x v x^{-1}+v$.

This gives three possibilities:

(i) $a \bar{c} \neq 0$ and $x$ is a scalar multiple of $a \bar{c}$,

(ii) $a=0$ and $\bar{c} v c=x v x^{-1}+v$ with $|c|=1$, giving (3) with $p=\bar{c}$ and $q=\hat{x}$,

(iii) $c=0$ and $\bar{a} x v x^{-1} a=x v x^{-1}+v$ with $|a|=1$, giving (3) with $p=\bar{a} \hat{x}$ and $q=\hat{x}$. 
In the case that $a \bar{c} \neq 0$ and $x$ is a scalar multiple of $a \bar{c}$, it follows that

$$
\begin{aligned}
\frac{1}{|a|^{2}|c|^{2}} \bar{a} a \bar{c} v c \bar{a} a+\bar{c} v c & =\frac{1}{|a|^{2}|c|^{2}} a \bar{c} v \bar{c} a+v, \\
\frac{|a|^{2}}{|c|^{2}} \bar{c} v c+\bar{c} v c & =\frac{1}{|a|^{2}|c|^{2}} a \bar{c} v \bar{c} a+v, \\
\frac{1}{|c|^{2}} \bar{c} v c & =\frac{1}{|a|^{2}|c|^{2}} a \bar{c} v \bar{c} a+v, \\
c^{-1} v c & =a \bar{c} v(a \bar{c})^{-1}+v .
\end{aligned}
$$

This gives (3) with $p=\hat{c}^{-1}$ and $q=\widehat{a \bar{c}}$. Solutions to (3) must obey $|\operatorname{Im}(p)| \geq \frac{1}{2}$ and $|\operatorname{Im}(q)| \geq \frac{\sqrt{3}}{2}$. This follows easily as a corollary to Proposition 4.1] see Remark 4.3. This gives the restrictions on the magnitude of the imaginary parts and covers all contingencies, (i), (ii) and (iii).

Consider the nature of a solution to condition (3) in general. Right multiplying by $p$, it follows that $p v=q v \bar{q} p+v p$. Taking the real part, since $\operatorname{Re}(p v)=\operatorname{Re}(v p)$ it follows that $\operatorname{Re}(v \bar{q} p q)=\operatorname{Re}(q v \bar{q} p)=0$. Thus $v \perp \operatorname{Im}(\bar{q} p q)$. Put $w=q v \bar{q}$. Note that $w \perp \operatorname{Im}(p)$. Then $p \bar{q} w q \bar{p}=\bar{q} w q+w$. So by the same logic, setting $p^{\prime}=p \bar{q}$ and $q^{\prime}=\bar{q}$, it follows that $w \perp \operatorname{Im}\left(q p \bar{q}^{2}\right)$, or rather $v \perp \operatorname{Im}(p \bar{q})$. Thus it follows that $v$ must be a scalar multiple of $v^{\prime}=\operatorname{Im}(\bar{q} p q) \times \operatorname{Im}(p \bar{q})$ provided $v^{\prime} \neq 0$, giving (a).

If not, then $\operatorname{Im}(p)$ and $\operatorname{Im}\left(q p \bar{q}^{2}\right)$ are linearly dependent. Hence

$$
\lambda \operatorname{Im}(p)=\operatorname{Im}((q p \bar{q}) \bar{q})=-\operatorname{Re}(q p \bar{q}) \operatorname{Im}(q)+\operatorname{Re}(q) \operatorname{Im}(q p \bar{q})-\operatorname{Im}(\operatorname{Im}(q p \bar{q}) \operatorname{Im}(q)) .
$$

Conjugating by $\bar{q}$ and rearranging,

$$
\operatorname{Im}(\operatorname{Im}(p) \operatorname{Im}(q))=\operatorname{Re}(q) \operatorname{Im}(p)-\operatorname{Re}(q) \operatorname{Im}(q)-\lambda \bar{q} \operatorname{Im}(p) q .
$$

Without loss put $q=e^{j \theta}$ and $\operatorname{Im}(p)=\sin (\eta)(\cos (\phi) i+\sin (\phi) j)$ to find

$$
\begin{aligned}
\sin (\eta) \cos (\phi) \sin (\theta) k= & \sin (\eta) \cos (\phi)(\cos (\theta)-\lambda \cos (2 \theta)) i \\
+ & (\cos (\theta) \sin (\eta) \sin (\phi)-\cos (\eta) \sin (\theta)-\lambda \sin (\eta) \sin (\phi)) j \\
& -\lambda \sin (\eta) \cos (\phi) \sin (2 \theta) k .
\end{aligned}
$$

Hence $\cos (\theta)=\lambda \cos (2 \theta)$ and $\sin \theta=-\lambda \sin (2 \theta)$, i.e. $e^{i \theta}=\lambda e^{-2 i \theta}$. Hence $\lambda= \pm 1$ and $e^{3 i \theta}= \pm 1$; hence $\theta=\frac{\pi}{3}$ or $\frac{2 \pi}{3}$. Recall that $p v \bar{p}=q v \bar{q}+v$; hence $|v|^{2}=$ $2|v|^{2}+2\langle q v \bar{q}, v\rangle$, i.e. $\langle q v \bar{q}, v\rangle /|v|^{2}=-\frac{1}{2}$, so the angle between $v$ and $q v \bar{q}$ is $\frac{2 \pi}{3}$. But since $\theta=\frac{\pi}{3}$ or $\frac{2 \pi}{3}$, it follows that $v \perp \operatorname{Im}(q)$. Since $v \perp \operatorname{Im}(\bar{q} p q)$, it follows that $v$ is a scalar multiple of

$$
v^{\prime \prime}=\operatorname{Im}(q) \times \operatorname{Im}(\bar{q} p q)=\bar{q} \operatorname{Im}(\operatorname{Im}(q) \operatorname{Im}(p)) q=\bar{q}(\operatorname{Im}(q) \times \operatorname{Im}(p)) q
$$

if $v^{\prime \prime} \neq 0$, that is, if $\operatorname{Im}(p)$ and $\operatorname{Im}(q)$ are linearly independent, giving (b).

If not, then both $\operatorname{Im}(p)$ and $\operatorname{Im}(q)$ are linearly dependent, or rather are multiples of unit length $\alpha \in \operatorname{Im}(\mathbb{H})$, and hence $p=e^{\alpha \frac{\pi}{6}}$ and $q= \pm e^{\alpha \frac{\pi}{3}}$ with $v \perp \alpha$, giving (c).

2.5. The $t \neq 0$ case.

Proposition 2.4. $Q \in \operatorname{Sp}(2)$ has zero planes, $\operatorname{span}\left\{\left(*, M^{1}\right),\left(*, M^{2}\right)\right\} \subset \mathcal{H}_{(e, Q)}$, of the form:

$$
M_{R}^{1}=\operatorname{diag}(-v, v), \quad M_{R}^{2}=\left(\begin{array}{cc}
0 & x \\
-\bar{x} & v
\end{array}\right)
$$


for $Q$ with $|a|^{2}=|c|^{2}=\frac{1}{2}$ precisely when

(i) $a \bar{c}$ is not real and $2 a \operatorname{Im}(a \bar{c}) \bar{c}-a \bar{c} \operatorname{Im}(a \bar{c})$ is purely imaginary and perpendicular to $a \bar{c}$, in which case one takes $v=\operatorname{Im}(a \bar{c})$ and $x=2 a \operatorname{Im}(a \bar{c}) \bar{c}-a \bar{c} \operatorname{Im}(a \bar{c})$;

(ii) $a= \pm c$ and $|\operatorname{Im}(c)| \geq \frac{1}{2 \sqrt{2}}$, in which case one takes $v$ such that $\operatorname{Re}(\operatorname{cv} \bar{c} v)=$ $-\frac{1}{4}|v|^{2}$. If $a=c$, then one takes $x=2 c v \bar{c}-\frac{1}{2} v$; if $a=-c$, then one takes $x=-2 c v \bar{c}+\frac{1}{2} v$.

Proof. Recall that a zero plane necessarily has the form (2). $x v x^{-1}$ and $v$ are linearly dependent; hence $x v x^{-1}= \pm v$. Suppose $x v x^{-1}=v$. Then $\bar{a} v a+\bar{c} v c=2 v$; however $|\bar{a} v a+\bar{c} v c| \leq\left(|a|^{2}+|c|^{2}\right)|v|=|v|$, giving a contradiction. Hence $x v x^{-1}=$ $-v$; thus it follows from the horizontal equation that $u_{L}^{1}=-\bar{a} v a+\bar{c} v c=0$. This is only possible if $|a|^{2}=|c|^{2}=\frac{1}{2}$, in which case $v a \bar{c}=a \bar{c} v$. Since $|b|^{2}=|d|^{2}=\frac{1}{2}$ and $b \bar{d}=-a \bar{c}$ it follows that $v_{L}^{1}=-\bar{b} v b+\bar{d} v d=0$, giving $\left[u_{L}^{1}, u_{L}^{2}\right]=\left[v_{L}^{1}, v_{L}^{2}\right]=0$. Since $x v x^{-1}=-v$ it follows that $\operatorname{Re}(x)=0$ and $x \perp v$. Then

$$
M_{L}^{2}=\operatorname{Ad}\left(Q^{-1}\right)\left(\begin{array}{cc}
0 & x \\
-\bar{x} & t v
\end{array}\right)=\left(\begin{array}{cc}
\bar{a} x c-\bar{c} \bar{x} a+t \bar{c} v c & * \\
* & \bar{b} x d-\bar{d} x b+t \bar{d} v d
\end{array}\right) .
$$

The horizontal condition now requires that $\bar{a} x c-\bar{c} \bar{x} a+t \bar{c} v c=t v$, or rather

$$
\bar{a} x c-\bar{c} \bar{x} a=t(v-\bar{c} v c) .
$$

$a\left(\right.$ (4) $\bar{c}$ yields $|a|^{2}|c|^{2} x-a \bar{c} \bar{x} a \bar{c}=t\left(a v \bar{c}-a \bar{c}|c|^{2} v\right)$ or $\frac{1}{4} x+a \bar{c} x a \bar{c}=t\left(a v \bar{c}-\frac{1}{2} a \bar{c} v\right)$, i.e. $x+p x p=t(4 a v \bar{c}-p v)$ where $p=2 a \bar{c}$ has $|p|=1$. Recall that $v a \bar{c}=a \bar{c} v$; thus $v p=p v$. Assume for $\operatorname{Im}(p) \neq 0$ that $v$ is a scalar multiple of $\operatorname{Im}(p)$. It follows that $x \perp \operatorname{Im}(p)$, and hence $x+p \bar{p} x=t(4 a v \bar{c}-p v)$, or rather $2 x=t(4 a v \bar{c}-2 a \bar{c} v)$. If $p= \pm 1$, substitution gives $2 x=t(4 a v \bar{c}-2 a \bar{c} v)$ again. Hence $x=t(2 a v \bar{c}-a \bar{c} v)$. With no loss of generality take $t=1$ to get

$$
x=2 a v \bar{c}-a \bar{c} v .
$$

Assume $a \bar{c}$ is not real. Without loss let $v=\operatorname{Im}(a \bar{c})$. The condition that $x=2 a v \bar{c}-$ $a \bar{c} v$ is purely imaginary and perpendicular to $v$ becomes that $2 a \operatorname{Im}(a \bar{c}) \bar{c}-a \bar{c} \operatorname{Im}(a \bar{c})$ is purely imaginary and perpendicular to $a \bar{c}$, giving (i).

It follows from Proposition 4.2 that when $a= \pm c$, (5) has solutions precisely when $|\operatorname{Im}(c)| \geq \frac{1}{2 \sqrt{2}}$; see also Remark 4.4. If $a=c$, then (5) becomes $x=2 c v \bar{c}-$ $|c|^{2} v=2 c v \bar{c}-\frac{1}{2} v$. If $a=-c$, then (15) becomes $x=-2 c v \bar{c}+|c|^{2} v=-2 c v \bar{c}+\frac{1}{2} v$. In both cases $\operatorname{Re}(x)=0$. The condition that $v$ is perpendicular to $x$ means that $v$ is restricted by $\langle c v \bar{c}, v\rangle=\frac{1}{4}|v|^{2}$, completing (ii).

\section{ESCHENBURG AND KERIN's METRIC}

Let $G=\operatorname{Sp}(2), H=\operatorname{Sp}(1)$ and $K=\operatorname{Sp}(1)^{2}$. Let $\varrho_{L}(p, q)=\operatorname{diag}(p, q), \varrho_{R}(q)=$ $\operatorname{diag}(q, 1)$ and $\varrho(q)=(q, q)$. By Proposition 1.5 we have that $G / / H$ is diffeomorphic to $H \times K \times G / / H \times H \times K$. Take the bi-invariant metric on $H \times K=\operatorname{Sp}(1)^{3}$ given by $s\langle,\rangle \oplus t(\langle,\rangle \oplus\langle\rangle$,$) . Observe that the metric induced by Proposition 1.5$ is isometric to Eschenburg and Kerin's metric of [3] via the equivariant isometry defined by the inverse on $\operatorname{Sp}(2), Q \mapsto Q^{-1}=Q^{*}$.

3.1. Horizontal vectors. With no loss of generality consider a coset representative of the form $P=(1,1,1, Q) \in H \times K \times G$.

Let $(\alpha, \beta, \gamma, M) \in \mathfrak{h} \oplus \mathfrak{k} \oplus T_{Q} G=T_{P}(H \times K \times G)$. Define $M_{L}$ and $M_{R}$ as previously. 
Proposition 3.1. The horizontal distribution at $(1,1,1, Q)$ is

$$
\mathcal{H}=\left\{\left(t^{-1} u_{L}, s^{-1} u_{R}, s^{-1} v_{R}, M\right) \in T_{P}(H \times K \times G): u_{L}=u_{R}+v_{R}\right\} .
$$

Proof. Let $\epsilon \in \operatorname{Im}(\mathbb{H})$ be arbitrary. Consider the field induced from $(0,0, \epsilon, 0)$, i.e. $(0,-\epsilon, 0, \operatorname{diag}(\epsilon, 0) Q)$. Hence the horizontal vector satisfies $s\langle-\epsilon, \beta\rangle+\left\langle\epsilon, u_{R}\right\rangle=0$, i.e. $\beta=s^{-1} u_{R}$. Likewise from the field $(0,0,0, \epsilon)$ we find that $\gamma=s^{-1} v_{R}$.

Consider the field induced from $(0, \epsilon, 0,0)$, i.e. $(-\epsilon, \epsilon, \epsilon, 0)$; the horizontal vector satisfies $t\langle-\epsilon, \alpha\rangle+s\langle\epsilon, \beta\rangle+s\langle\epsilon, \gamma\rangle=0$, i.e. $\alpha=t^{-1} s(\beta+\gamma)=t^{-1}\left(u_{R}+v_{R}\right)$.

Consider the field induced from $(\epsilon, 0,0,0)$, i.e. $(\epsilon, 0,0, Q \operatorname{diag}(-\epsilon, 0))$; the horizontal vector satisfies $t\langle\epsilon, \alpha\rangle+\left\langle u_{L},-\epsilon\right\rangle=0$, i.e. $\alpha=t^{-1} u_{L}$. Hence $u_{L}=u_{R}+v_{R}$.

3.2. Zero planes. It follows from Proposition 1.2 that zero planes, $\operatorname{span}\left\{M^{1}, M^{2}\right\}$, are characterized by the conditions $\left[u_{R}^{1}, u_{R}^{2}\right]=0,\left[v_{R}^{1}, v_{R}^{2}\right]=0$, and $\left[M_{R}^{1}, M_{R}^{2}\right]=$ 0 and $\left[u_{L}^{1}, u_{L}^{2}\right]=0$ with $u_{L}^{i}=u_{R}^{i}+v_{R}^{i}$. The Wilhelm metric has in addition $\left[v_{L}^{1}, v_{L}^{2}\right]=0$. But from the earlier analysis of the Wilhelm metric, given the first three conditions one can assume without loss either (1) or (2), and in either case the third condition $\left[u_{L}^{1}, u_{L}^{2}\right]=0$ then implies the fourth condition that $\left[v_{L}^{1}, v_{L}^{2}\right]=0$. So in fact both Eschenburg and Kerin's metric and Wilhelm's metric have equivalent zero plane loci.

\section{Structure of the Zero LOCUS}

Propositions 2.3 and 2.4 allow one to determine if a given $[Q] \in M$ is in the zero locus. In contrast a useful parametrisation follows from Propositions 4.1 and 4.2 .

Remark 4.1. Proposition 4.1 addresses the general form of a solution to the condition (3) in Proposition 2.3.

Proposition 4.1. The solutions, $p, q \in \operatorname{Sp}(1)$ and $v \in \operatorname{Im}(\mathbb{H})$ with $|v|=1$ to

$$
p v \bar{p}=q v \bar{q}+v
$$

are parametrised by $(p, q, v)=\left(e^{w \frac{\pi}{6}} e^{v \theta}, e^{w \frac{\pi}{3}} e^{v \phi}, v\right)$, where $\theta, \phi \in \mathbb{R}$ and $w \in \operatorname{Im}(\mathbb{H})$ with $|w|=1$ and $w \perp v$ and $v \in \operatorname{Im}(\mathbb{H})$ with $|v|=1$.

Proof. Write $q v \bar{q}=-(q v \bar{q} v) v$. Note that $\langle-q v \bar{q} v, v\rangle=\operatorname{Re}(q v \bar{q})=0$ since $\operatorname{Re}(v)=$ 0 . Note also $|-q v \bar{q} v|=1$. Recall that $\langle q v \bar{q}, v\rangle=-\frac{1}{2}$. Thus $\operatorname{Re}(-q v \bar{q} v)=$ $-\frac{1}{2}$. Hence $-q v \bar{q} v=e^{w \frac{2 \pi}{3}}$ for $w= \pm \widehat{\operatorname{Im}(q v \bar{q} v)}$. It follows that $q v \bar{q}=e^{w \frac{2 \pi}{3}} v=$ $e^{w \frac{\pi}{3}} v e^{-w \frac{\pi}{3}}$. Hence $\left(e^{-w \frac{\pi}{3}} q\right) v\left(e^{-w \frac{\pi}{3}} q\right)^{-1}=v$ and thus $e^{-w \frac{\pi}{3}} q=e^{v \phi}$, or rather $q=e^{w \frac{\pi}{3}} e^{v \phi}$.

$q v \bar{q}+v=\left(-\frac{1}{2}+\frac{\sqrt{3}}{2} w\right) v+v=\left(\frac{1}{2}+\frac{\sqrt{3}}{2} w\right) v=e^{w \frac{\pi}{3}} v=e^{w \frac{\pi}{6}} v e^{-w \frac{\pi}{6}}$. So $p v \bar{p}=$ $e^{w \frac{\pi}{6}} v e^{-w \frac{\pi}{6}}$, or rather $e^{-w \frac{\pi}{6}} p v \bar{p} e^{w \frac{\pi}{6}}=v$. Hence $e^{-w \frac{\pi}{6}} p=e^{v \theta}$, or rather $p=e^{w \frac{\pi}{6}} e^{v \theta}$. Substitution reveals that $(p, q, v)=\left(e^{w \frac{\pi}{6}} e^{v \theta}, e^{w \frac{\pi}{3}} e^{v \phi}, v\right)$ solves $p v \bar{p}=q v \bar{q}+v$.

Remark 4.2. Proposition 4.2 addresses the general form of a solution to condition (4) in Proposition 2.4. Equation (6) in Proposition 4.2 below is equivalent to (4) with $a=\frac{1}{\sqrt{2}} p, c=\frac{1}{\sqrt{2}} q$ and $t=1$.

Proposition 4.2. The solutions $p, q \in \operatorname{Sp}(1), v, x \in \operatorname{Im}(\mathbb{H})$ and $x \perp v$ with $|v|=1$ to

$$
\bar{p} x q+\bar{q} x p=2 v-\bar{q} v q
$$


such that $v p \bar{q}=p \bar{q} v$ are parametrised by

$$
(p, q, x, v)=\left(e^{v \theta} e^{w \frac{\pi}{6}} e^{v \phi}, e^{w \frac{\pi}{6}} e^{v \phi}, \frac{\sqrt{3}}{2} e^{v \theta} w v, v\right),
$$

where $v, w \in \operatorname{Im}(\mathbb{H})$ with $|v|=|w|=1$ and $v \perp w$ and $\theta, \phi \in \mathbb{R}$.

Proof. $p(\underline{6}) \bar{q}$ is $x+p \bar{q} x p \bar{q}=2 p v \bar{q}-p \bar{q} v$. It follows that $2 x+p \bar{q} v=2 p v \bar{q}$. Now $p \bar{q}=e^{v \theta}$ since $v p \bar{q}=p \bar{q} v$. Hence $p \bar{q} v \perp x$ since both $x \in \operatorname{Im}(\mathbb{H})$ and $x \perp v$. This in conjunction with the fact that $x+\frac{1}{2} p \bar{q} v=p v \bar{q}$ implies $|x|^{2}+\frac{1}{4}=1$. Hence $|x|^{2}=\frac{3}{4}$. So $x=\frac{\sqrt{3}}{2} \hat{x}$. Thus

$$
p v \bar{q}=\frac{1}{2} e^{v \theta} v+\frac{\sqrt{3}}{2} \hat{x} .
$$

Let $r=\frac{1}{2} e^{v \theta}-\frac{\sqrt{3}}{2} \hat{x} v$. Then $p v \bar{q}=r v$. Hence $e^{v \theta} q v \bar{q}=r v$. Thus $q v \bar{q}=$ $\left(\frac{1}{2}-\frac{\sqrt{3}}{2} e^{-v \theta} \hat{x} v\right) v=e^{w \frac{\pi}{3}} v=e^{w \frac{\pi}{6}} v e^{-w \frac{\pi}{6}}$, where $w=-e^{-v \theta} \hat{x} v$. Therefore $e^{-w \frac{\pi}{6}} q v\left(e^{-w \frac{\pi}{6}} q\right)^{-1}=v$. Hence $e^{-w \frac{\pi}{6}} q=e^{v \phi}$, or rather $q=e^{w \frac{\pi}{6}} e^{v \phi}$. Consequently $p=e^{v \theta} e^{w \frac{\pi}{6}} e^{v \phi}$ and $\hat{x}=e^{v \theta} w v$. Again substitution reveals that such data gives a solution to (6).

Remark 4.3. Note in Proposition 4.1 that $p, q$ are such that

$$
\operatorname{Im}(p)=\frac{1}{2} e^{-v \theta} w+\frac{\sqrt{3}}{2} \sin (\theta) v, \quad \operatorname{Im}(q)=\frac{\sqrt{3}}{2} e^{-v \phi} w+\frac{1}{2} \sin (\phi) v .
$$

Thus $|\operatorname{Im}(p)|^{2}=\frac{1}{4}+\frac{3}{4} \sin ^{2}(\theta)$ and $|\operatorname{Im}(q)|^{2}=\frac{3}{4}+\frac{1}{4} \sin ^{2}(\phi)$.

Remark 4.4. Note in Proposition 4.2 that $p$ is such that

$$
\operatorname{Im}(p)=\frac{\sqrt{3}}{2} v \sin (\theta+\phi)+\frac{1}{2} e^{v(\theta-\phi)} w .
$$

Hence $|\operatorname{Im}(p)|^{2}=\frac{1}{4}+\frac{3}{4} \sin ^{2}(\theta+\phi)$. Similarly it follows that $|\operatorname{Im}(q)|^{2}=\frac{1}{4}+\frac{3}{4} \sin ^{2}(\phi)$.

Proposition 4.3. Any $[Q] \in M=G / / H$ in the zero locus up to the isometric action of $\mathrm{Sp}(1)$ via

$$
r \odot[Q] \equiv\left[Q\left(\begin{array}{ll}
1 & 0 \\
0 & \bar{r}
\end{array}\right)\right]
$$

can be represented by $Q \in \operatorname{Sp}(2)$ of one of the forms

$$
\begin{gathered}
Q=\left(\begin{array}{cc}
e^{j \frac{\pi}{3}} e^{i(\phi-\theta)} e^{-j \frac{\pi}{6}} \cos (\psi) & -\sin (\psi) \\
e^{-i \theta} e^{-j \frac{\pi}{6}} \sin (\psi) & e^{-i \phi} e^{-j \frac{\pi}{3}} \cos (\psi)
\end{array}\right), \\
Q=\frac{1}{\sqrt{2}}\left(\begin{array}{cc}
e^{i \theta} e^{j \frac{\pi}{6}} e^{i \phi} & -1 \\
e^{j \frac{\pi}{6}} e^{i \phi} & e^{-i \theta}
\end{array}\right),
\end{gathered}
$$

where $\theta, \phi, \psi \in \mathbb{R}$.

Proof. The proof splits into the $t=0$ case and the $t \neq 0$ case.

In the $t=0$ case since $|a|^{2}+|c|^{2}=1$ put $a=\hat{a} \cos (\psi)$ and $c=\hat{c} \sin (\psi)$. Generically from Propositions 2.3 and 4.1 it follows that $\hat{c}=\bar{p}=e^{-v \theta} e^{-w \frac{\pi}{6}}$ and $\hat{a}=\widehat{a} \bar{c} \hat{c}=q \bar{p}=e^{w \frac{\pi}{3}} e^{v(\phi-\theta)} e^{-w \frac{\pi}{6}}$. Without loss assume $b=-\sin (\psi)$ since $b \bar{r}$ is real for appropriate $r$. It follows that $b \bar{d}=-a \bar{c}=-\cos (\psi) \sin (\psi) q$. Hence $d=\cos (\psi) \bar{q}=\cos (\psi) e^{-v \phi} e^{-w \frac{\pi}{3}}$. 
In the $t \neq 0$ case, $|a|^{2}=|c|^{2}=\frac{1}{2}$. Let $a=\frac{1}{\sqrt{2}} p$ and $c=\frac{1}{\sqrt{2}} q$ and as before let $b=-\frac{1}{\sqrt{2}}$ be real. Then $d=\frac{1}{\sqrt{2}} q \bar{p}$. It follows from Propositions 2.4 and 4.2 that $a=\frac{1}{\sqrt{2}} e^{v \theta} e^{w \frac{\pi}{6}} e^{v \phi}, c=\frac{1}{\sqrt{2}} e^{w \frac{\pi}{6}} e^{v \phi}$ and $d=\frac{1}{\sqrt{2}} e^{-v \theta}$.

Let $\left(\begin{array}{cc}a^{\prime} & b^{\prime} \\ c^{\prime} & d^{\prime}\end{array}\right)=s \cdot Q=\left(\begin{array}{cc}s a \bar{s} & s b \\ s c \bar{s} & s d\end{array}\right)$ and $\left(v^{\prime}, w^{\prime}\right)=(s v \bar{s}, s w \bar{s})$.

In the $t=0$ case, $Q$ takes the form $\left(\begin{array}{cc}e^{w \frac{\pi}{6}} e^{v(\phi-\theta)} e^{-w \frac{\pi}{6}} \cos (\psi) & * \\ e^{-v \theta} e^{-w \frac{\pi}{6}} \sin (\psi) & *\end{array}\right)$. Thus

$$
\left(\begin{array}{ll}
a^{\prime} & b^{\prime} \\
c^{\prime} & d^{\prime}
\end{array}\right)=\left(\begin{array}{cc}
s e^{w \frac{\pi}{6}} e^{v(\phi-\theta)} e^{-w \frac{\pi}{6}} \cos (\psi) \bar{s} & * \\
s e^{-v \theta} e^{-w \frac{\pi}{6}} \sin (\psi) \bar{s} & *
\end{array}\right)=\left(\begin{array}{cc}
e^{w^{\prime} \frac{\pi}{6}} e^{v^{\prime}(\phi-\theta)} e^{-w^{\prime} \frac{\pi}{6}} \cos (\psi) & * \\
e^{-v^{\prime} \theta} e^{-w^{\prime} \frac{\pi}{6}} \sin (\psi) & *
\end{array}\right) .
$$

Similarly in the $t \neq 0$ case $Q$ takes the form $\frac{1}{\sqrt{2}}\left(\begin{array}{cc}e^{v \theta} e^{w \frac{\pi}{6}} e^{v \phi} & * \\ e^{v \frac{\pi}{6}} e^{w \phi} & *\end{array}\right)$. Thus

$$
\left(\begin{array}{cc}
a^{\prime} & b^{\prime} \\
c^{\prime} & d^{\prime}
\end{array}\right)=\frac{1}{\sqrt{2}}\left(\begin{array}{cc}
s\left(e^{v \theta} e^{w \frac{\pi}{6}} e^{v \phi}\right) \bar{s} & * \\
s\left(e^{v \frac{\pi}{6}} e^{w \phi}\right) \bar{s} & *
\end{array}\right)=\frac{1}{\sqrt{2}}\left(\begin{array}{cc}
e^{v^{\prime} \theta} e^{w^{\prime} \frac{\pi}{6}} e^{v^{\prime} \phi} & * \\
e^{v^{\prime} \frac{\pi}{6}} e^{w^{\prime} \phi} & *
\end{array}\right) .
$$

It follows from the transitivity of the action on the two orthonormal frames that one may assume without loss that $(v, w)=(i, j)$ in either case from the outset.

Remark 4.5. The two parametrised components of the zero locus in Proposition 4.3 are 6-dimensional and 5-dimensional in $M=G / / H$ for forms (7) and (8) respectively. Since $M=G / / H$ is 7-dimensional the manifold has almost positive sectional curvature.

\section{REFERENCES}

[1] Cheeger, J., Some examples of manifolds of non-negative curvature. J. Differential Geom. 8 (1973), 623-628. MR0341334(49:6085)

[2] Eschenburg, J.-H., Freie isometrische Aktionen auf kompakten Lie-Gruppen mit positiv gekrümmten Orbiträumen. Schr. Math. Inst. Münster 32 (1984). MR.758252 (86a:53045)

[3] Eschenburg, J.-H.; Kerin, M. Almost positive curvature on the Gromoll-Meyer sphere. Proc. Amer. Math. Soc. 136 (2008), 3263-3270. MR2407092 (2009a:53061)

[4] Gromoll, D., Meyer, W., An exotic sphere with non-negative sectional curvature. Ann. of Math. (2) 100 (1974), 401-406. MR0375151(51:11347)

[5] O'Neill, B. The fundamental equations of a submersion. Michigan Math. J. 13 (1966), 459-469. MR0200865 (34:751)

[6] Wilhelm, F. An exotic sphere with positive curvature almost everywhere. J. Geom. Anal. 11 (2001), 519-560. MR1857856 (2002f:53056)

[7] Wilking, B., Manifolds with positive sectional curvature almost everywhere. Invent. Math. 148 (2002), 117-141. MR.1892845 (2003a:53049)

Department of Mathematics, University of California, Riverside, Riverside, CaliFORNIA 92521

Current address: Department of Mathematics, University of California, Los Angeles, Box 951555, Los Angeles, California 90095-1555

E-mail address: owend@ucr.edu 